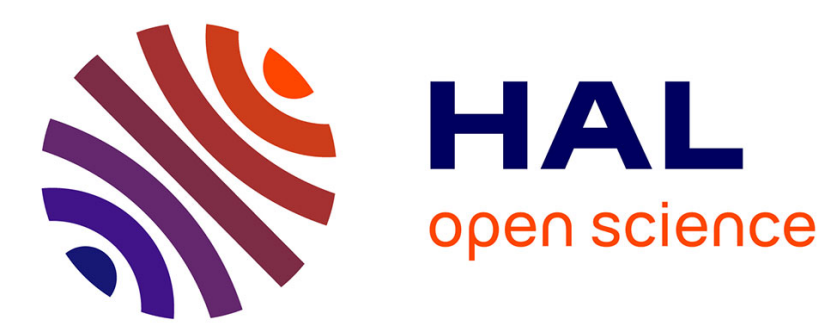

\title{
PEO-PPO-PEO triblock copolymer in aqueous solution. Micelle formation and crystallization
}

\author{
K. Mortensen
}

\section{To cite this version:}

K. Mortensen. PEO-PPO-PEO triblock copolymer in aqueous solution. Micelle formation and crystallization. Journal de Physique IV Proceedings, 1993, 03 (C8), pp.C8-157-C8-160. 10.1051/jp4:1993830 . jpa-00252261

\section{HAL Id: jpa-00252261 https://hal.science/jpa-00252261}

Submitted on 1 Jan 1993

HAL is a multi-disciplinary open access archive for the deposit and dissemination of scientific research documents, whether they are published or not. The documents may come from teaching and research institutions in France or abroad, or from public or private research centers.
L'archive ouverte pluridisciplinaire HAL, est destinée au dépôt et à la diffusion de documents scientifiques de niveau recherche, publiés ou non, émanant des établissements d'enseignement et de recherche français ou étrangers, des laboratoires publics ou privés. 


\title{
PEO-PPO-PEO triblock copolymer in aqueous solution. Micelle formation and crystallization
}

\author{
K. MORTENSEN
}

Physics Department, Ris $\emptyset$ National Laboratory, 4000 Roskilde, Denmark

\begin{abstract}
The phase behavior of poly(ethylene oxide) - poly(propylene oxide) - poly(ethylene oxide), PEO-PPO-PEO, tri-block copolymers dissolved in water has been studied using small-angle neutron scattering, using a Couette device for shear alignments. The structural properties has been studied as a function of polymer concentration and temperature. At low-temperature and low polymer concentrations the unimers are dissolved as individual Gaussian chains. At temperatures close to ambient, the hydrophobic nature of PPO causes aggregation of the polymers into spherical micelles. The aggregation number is relative small close to the critical micellation temperature, and increases markedly with temperature. The micellar volume fraction increases roughly linearly with temperature, until either a saturation is reached, where all polymers are part of a micelle, or the volume fraction reaches the critical value for hard-sphere crystallization. At higher temperatures the aggregates transform from spherical form to rod-like micelles, causing first melting of the cubic structure, and subsequently crystallization into a phase of hexagonally ordered rods.
\end{abstract}

\section{INTRODUCTION}

Block copolymers composed of poly(ethylene oxide) and poly(propylene oxide) have attracted increasing attention during the last few years [1-9]. This is partly due to their wide range of applications as non-ionic surface active agents, and partly because of their unique behavior which is of fundamental interest in physics [4-6].

The association properties of triblock copolymers $\mathrm{E}_{m} \mathrm{P}_{n} \mathrm{E}_{m}$ in aqueous solutions have been studied by various techniques, and the thermally reversible gelation, displayed in some cases, has been the subject of many investigations $[2,3,4]$. Recently it was shown by neutron scattering that the gel state is a crystalline phase of $\mathrm{BCC}$ ordered spherical micelles [4-6].

Different $\mathrm{E}_{m} \mathrm{P}_{n} \mathrm{E}_{m}$ triblock copolymers with varying $m$ and $n$ have been studied [1-9]. In many cases there is association into micelles at elevated temperatures, but other forms of aggregate may also form [7]. In the present letter, we concentrate on a single system, forming spherical micelles close to ambient temperatures, and rod-like micelles at higher temperatures.

\section{MATERIAL}

The tri-block copolymer, poly(ethylene oxide) - poly(propylene oxide) - poly(ethylene oxide),

$$
\mathrm{H}\left[\mathrm{OCH}_{2} \mathrm{CH}_{2}\right]_{m} \quad\left[\mathrm{OCH}_{2} \mathrm{CH}\left(\mathrm{CH}_{3}\right)\right]_{n} \quad\left[\mathrm{OCH}_{2} \mathrm{CH}_{2}\right]_{m} \mathrm{H}
$$


with $n=39$, and $m=27$ abbreviated P85 was obtained from Serva AG, Heidelberg, Germany. The triblock copolymers were dissolved in water at $5^{\circ} \mathrm{C}$, at which they form a transparent, homogeneous solution. Deuterated water, $\mathrm{D}_{2} \mathrm{O}$, was used in order to get good contrast and low background for the neutron scattering experiments.

\section{SANS}

Small-angle neutron scattering experiments were performed using the Ris $\varnothing$-SANS facility, which is a flexible instrument covering scattering vectors from 0.002 to $0.5 \AA^{-1}$, with variable neutron wavelength resolution. The samples were mounted in sealed quartz containers (Suprasil from Hellma, FRG), with $2 \mathrm{~mm}$ flight path. For shear alignments, the samples were mounted in a Couette cell with $0.5 \mathrm{~mm}$ between the inner rotor and outer stator, which were both made of niobium.

The results presented below were obtained using neutrons with $6 \AA$ wavelength, with sample-todetector distances of $3 \mathrm{~m}$, giving scattering vectors in the range 0.01-0.1 $\AA^{-1}$, where the scattering vector $\mathbf{q}$ is given by the scattering angle $\theta$ and the neutron wavelength $\lambda:|\mathbf{q}|=q=4 \pi / \lambda \cdot \sin (\theta / 2)$. The neutron wavelength resolution used was $\Delta \lambda / \lambda=0.18$, and the neutron beam collimation was determined by the pinhole sizes of $16 \mathrm{~mm}$ and $7 \mathrm{~mm}$ diameter at source and sample position respectively, and collimation lengths of $3 \mathrm{~m}$. The smearing induced by the wavelength spread, the collimation and the detector resolution was included in the data analysis discussed below, using Gaussian approximations for the different terms.

\section{RESULTS and DISCUSSION}

The scattering patterns show the typical characteristics: at low temperatures, the scattering function shows relatively weak $q$-dependence and the absolute intensity is small. As the temperature increases, the intensity increases and the $q$-dependence becomes stronger, revealing aggregation of copolymers. At even higher temperatures, the scattering function of the copolymer solutions is increasingly dominated by a pronounced peak, revealing important spatial correlation between neighboring aggregates.

\section{Gaussian Unimers}

The low temperature neutron scattering patterns of relative small intensity have previously been shown to be consistent with fully dissolved Gaussian copolymer molecules [8]. For P85, we found a radius of gyration equal $17 \AA$.

\section{Micellar Aggregates}

At temperatures close to ambient, the poly(propylene-oxide) part of the polymer chain is no longer soluble in water. The resulting amphiphilic character of $\mathrm{E}_{m} \mathrm{P}_{n} \mathrm{E}_{m}$ tri-block copolymers leads to formation of various aggregates, depending on the degree of polymerization of the blocks. For P85 the scattering patterns reveal the formation of spherical micelles over a wide temperature regime $[5,8]$, i.e. the aggregates have the form of a core presumably dominated by propylene oxide blocks and which is surrounded by a corona of hydrated ethylene oxide sub-chains.

As the temperature, or the concentration, is increased the scattering function becomes increasingly dominated by a pronounced correlation peak which reveals significant interactions between neighboring micelles. In the high- $q$ range well beyond the correlation peak, on the other hand, the scattering function remains effectively unchanged indicating that the changes in the observed scattering function primarily reflect the increasing density of the micelles and the characteristic form of the individual micelles is relatively unaffected by increasing the temperature or the concentration, as seen in Fig. 1. 

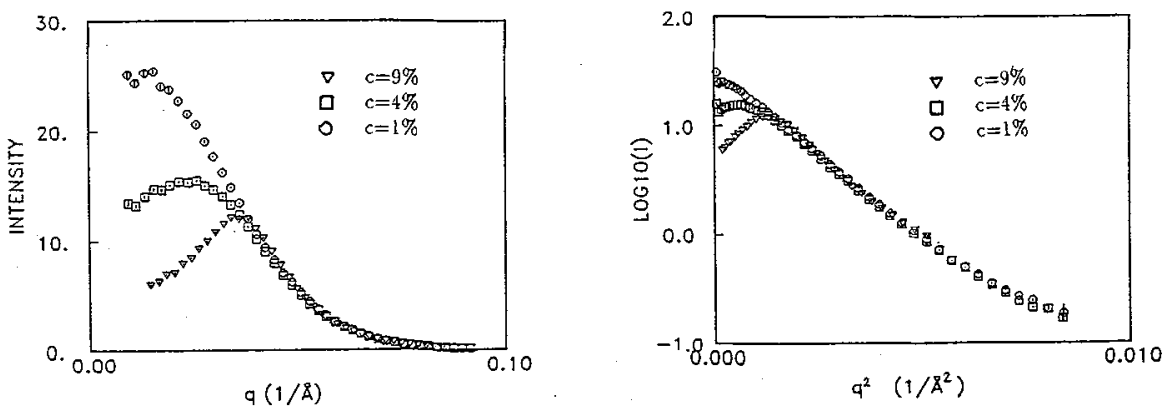

Fig. 1. Scattering function normalized to polymer concentratin of 1,4 and $9 \%$ P85 as obtained at $T=40^{\circ} \mathrm{C}$, plotted as $I$ vs $q$ and $\log (I)$ vs. $q^{2}$

The experimental scattering function has in this regime of marked inter-particle correlation been analyzed assuming the form of the scattering function:

$$
I(q)=\Delta \rho^{2} \cdot n \cdot P(q) \cdot S(q)
$$

where $\Delta \rho^{2}$ is the contrast factor and $n$ is the number density of scatterers. $P(q)$ is the particle formfactor, and $S(q)$ is the structure factor describing the inter-particle interferences. In the analysis, we have used the formfactor of dense spheres and the structure factor was described using the PercusYevick approach with a hard-sphere interaction potential. In Fig. 2 is shown the resulting core-radius, as obtained using this analysis for a wide range of concentrations of P85 in water.
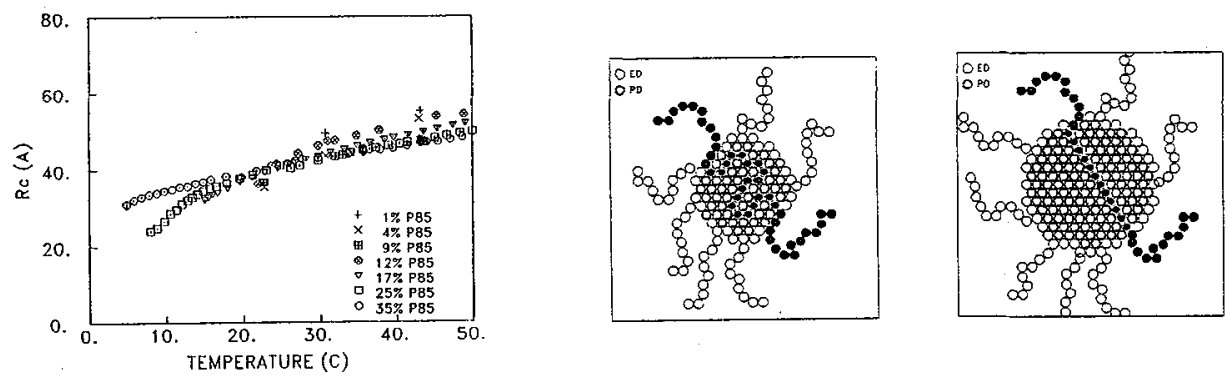

Fig. 2. The core radius of P85 micelles dissolved in water, as obtained from hard-sphere Percus Yevisk analysis of the SANS data, and model of the micelles at intermediate temperatures, and at temperature just below the temperature of transition from spherical to rodlike micelle

The micellar core-radius, $R_{c}$, (and the hard-sphere radius, $R_{h s}$ ), appear to be essentially independent of polymer concentration, but show significant increase with increasing temperatures. It is especially interesting to note that close to the highest temperatures, where spherical micelles are the stable form for aggregates, the core diameter is close to the size of a fully extended PPO-chain, as shown schematically in Fig. 2 for the polymer chain going through the micellar center. This is entropically a very unfavorable conformation and may be the driving force for the form-transformation of the micelles from spherical to prolate ellipsoidal, and subsequent rodlike form.

At low temperatures, the micellar hard-sphere volume fraction approaches zero, indicating that all copolymers are dissolved as individual unimers. Above a critical micellation temperature $\left(T_{c m 1}\right)$, the volume fraction appears roughly linear in temperature until a saturated value, $\phi_{o}$, is reached at $T_{c m 2}$. Within the temperature range, $T_{c m 1}-T_{c m 2}$, micelles are in thermodynamic equilibrium with the a pool of unimers. As pointed out in ref. [8] it is interesting to note that to a good approximation the limiting volume fraction, $\phi_{o}$, increases linearly with polymer concentration. This is in agreement with expectations if the limiting micelle concentration corresponds to the situation where all unimers have aggregated into micelles. 
It was shown in [8] that, to a very good approximation, the micellar core can be considered as consisting of completely dehydrated propylene oxide units, covered by a single mono-layer of ethylene oxide. In a numerical study Linse and Malmsten have shown a somewhat similar behavior, although they also found that some $\mathrm{EO}$ is present inside the core and that some $\mathrm{PO}$ monomers are present in the corona [9]. Neglecting the single EO-layer, which is anyway small compared to $R_{c}$, we then find that to a good approximation the aggregation number scales linearly with the third power of the core radius, $R_{c}: N \cdot n \cdot V_{P}=\frac{4}{3} \pi R_{c}^{3}$, where $V_{P}$ is the $\mathrm{PPO}$-monomer volume, and $N$ is the aggregation number.

For polymer concentrations above approximately $20 \%$, the limiting value of the micellar volume fraction is no longer linear with the polymer concentration, but remains at the value $\phi=0.53$ characteristic for soft Body Centered Cubic phases. The P85-micelles crystallize on a BCC lattice, for polymer concentrations above $20 \%$ and above a temperature determined by the micellar volume fraction. In the scattering pattern, however, only a very little change can be detected at the crystallization temperature $T_{c}$. P85 shows, in opposition to several other EPE-systems like F87, F88, F127, normally no texture in the Debye-Scherrer ring, and in the powder form, higher order reflections is only hardly detectable due to the micellar formfactor. In the order to prove the cubic crystalline nature, aligned samples is needed.
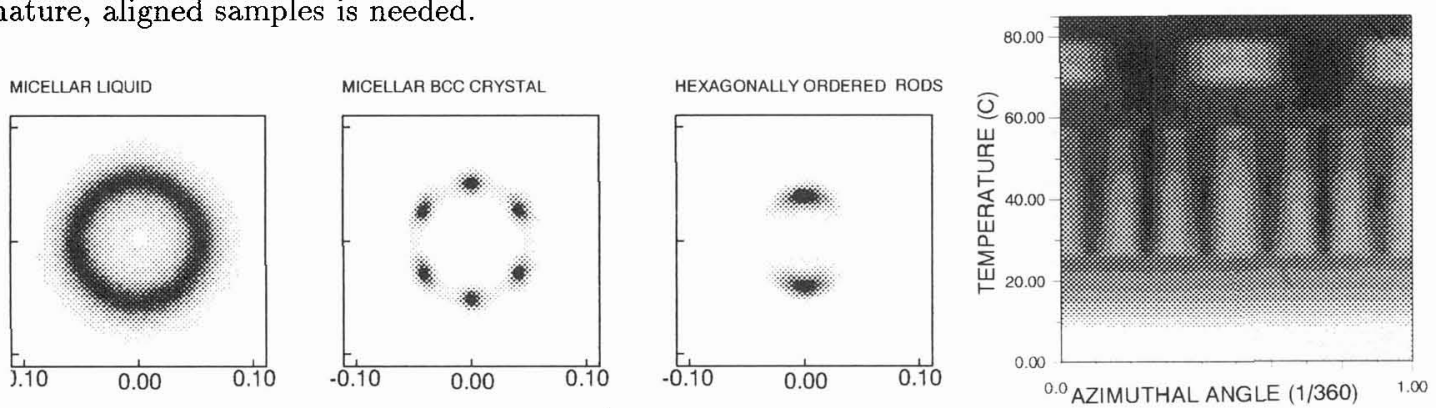

Fig. 3. Two dimensional scattering pattern of P85, and Contour plot in the azimuthal angle and temperature plane.

It appear, that PEO-PPO-PEO micellar crystals align surprisingly effective upon exposure to a shear field [4]. We have therefore made extended studies using both a Couette type of shear cell, and shear between two plane-parallel plates. In opposition to block copolymer melts [10], the orderdisorder transition temperature of P85 in water does not any marked shear dependence. In Fig. 3 is shown characteristic patterns, clearly revealing the crystalline structure. The $\mathrm{BCC}$ nature have been proven on samples sheared between two parallel plates. A very effective way to present the scattering patterns is a contour plot of azimuthal scan through the two-dimensional scattering pattern of Bragg peaks, as shown in Fig. 3.

\section{References}

[1] Zhou, Z; Chu, B.; Macromolecules 20 (1987) 3089; J. Colloid. Interface Sci. 126 (1988) 171.

[2] Wanka, G.; Hoffmann, H.; and Ulbricht, W., Colloid Polym. Science 268 (1990) 101.

[3] Brown, W.; Schillen, K.; Almgren, M.; Hvidt, S.; Bahadur, P., J.Phys.Chem. 95 (1991) 1850.

[4] Mortensen, K.; Brown, W.; and Nordén, B., Phys. Rev. Letters 19 (1992), 2340.

[5] Mortensen, K., Europhys. Letters 19 (1992) 599.

[6] Mortensen, K., Prog. Colloid Polym. Science 91 (1993 69.

[7] Mortensen, K., and Pedersen, J.S. Macromolecules 26 (1993) 805

[8] Mortensen, K., and Brown, W. Macromolecules 1993, submitted.

[9] Linse, P., Malmsten, M., Macromolecules 25 (1992) 5434.

[10] Bates, F., Koppi, K., Almdal, K., and Mortensen, K., To be published. 